\title{
Incommensurate Smectic Order at the Free Surface in the Nematic Phase of $\backslash(4-\mathrm{N}-H e p t y l p h e n y l-4 '-14 "-$ Nitrobenzoyloxy)Benzoate (DB7NO_2J)
}

\section{Citation}

Ocko, B. M., Peter S. Pershan, C. R. Safinya, and L. Y. Chiang. 1987. Incommensurate smectic order at the free surface in the nematic phase of $\backslash(4-n$-heptylphenyl-4'-(4"nitrobenzoyloxy)benzoate (DB7NO_2J). Physical Review A 35(4): 1868-1872.

\section{Published Version}

doi:10.1103/PhysRevA.35.1868

\section{Permanent link}

http://nrs.harvard.edu/urn-3:HUL.InstRepos:10357480

\section{Terms of Use}

This article was downloaded from Harvard University's DASH repository, and is made available under the terms and conditions applicable to Other Posted Material, as set forth at http:// nrs.harvard.edu/urn-3:HUL.InstRepos:dash.current.terms-of-use\#LAA

\section{Share Your Story}

The Harvard community has made this article openly available.

Please share how this access benefits you. Submit a story.

\section{Accessibility}




\title{
Incommensurate smectic order at the free surface in the nematic phase of 4- $n$-heptylphenyl-4'-(4"-nitrobenzoyloxy)benzoate (DB7NO $\left.{ }_{2}\right)$
}

\author{
B. M. Ocko and P. S. Pershan \\ Division of Applied Sciences, Harvard University, Cambridge, Massachusetts 02138 \\ C. R. Safinya and L. Y. Chiang \\ Corporate Research Science Laboratories, Exxon Research and Engineering Company, Annandale, New Jersey 08801
}

(Received 21 August 1986)

\begin{abstract}
We report $\mathrm{x}$-ray reflectivity measurements on the free surface of 4 - $n$-heptylphenyl-4' $-\left(4^{\prime \prime}\right.$ nitrobenzoyloxy)benzoate $\left(\mathrm{DB} 7 \mathrm{NO}_{2}\right)$ at the nematic to smectic- $A$ phase transition, $T_{N A}=99.9^{\circ} \mathrm{C}$. The free surface in the nematic phase exhibits smecticlike ordering at two $q$ vectors, one which is commensurate with the smectic- $\boldsymbol{A}$ monolayer $q$ vector $q_{2}$. The other $q$ vector is incommensurate corresponding to ordering at $\sim 0.59 q_{2}$. The commensurate peak constructively interferes with the air-liquid interface while the incommensurate peak destructively interferes. These results are compared with bulk-phase $\mathrm{x}$-ray scattering measurements.
\end{abstract}

Smectic liquid-crystal mesophases are layered phases which exhibit liquid order within the layers. For the smectic- $\boldsymbol{A}$ phase, which is the least-ordered smectic phase, the molecules are aligned along the layer normals with only short-range in-plane liquid order. Mesogens which have a weak dipole moment tend to form monolayer phases $\left(\operatorname{Sm} A_{1}\right)$, where the molecules within a layer are randomly up and down and the layer spacing $d_{2}$ is of the order of the molecular length $L$. On the other hand, molecules which have strong dipole moments form bilayer antiferroelectric phases $\left(\operatorname{Sm} A_{2}\right.$ or $\left.\operatorname{Sm} A_{d}\right)$ where the dipoles are preferentially up or down with a bilayer length less than or equal to $2 L$. For the $\operatorname{Sm} A_{2}$ phase the layer spacing $d_{1}=2 L$. This competition for ordering at two length scales is sometimes manifested by novel phase behavior, ${ }^{1}$ including phases transitions between $\operatorname{Sm} A_{1}$ and $\operatorname{Sm} A_{2}{ }^{2}$ coexisting phases with competing length scales, ${ }^{3,4}$ smectic phases with a long-wavelength in-plane modulation $(\mathrm{Sm} \widetilde{A}$ and $\operatorname{SmC}),{ }^{5,6}$ and multiply reentrant phases. ${ }^{7-9}$

The Landau-de Gennes model has provided a theoretical starting point for the nematic to smectic- $A$ transition where the order parameter $\Psi_{2}(r)$ describes the smectic mass density modulation at $q_{2}=2 \pi / d_{2}$. Prost and Barois ${ }^{1}$ have extended this model to include the antiferroelectric order parameter, $\Psi_{1}$ and a coupling term $\Psi_{1}^{2} \Psi_{2}^{*}$ which favors commensurability. In the nematic phase the coupling is usually weak and fluctuations of both $\Psi_{1}$ and $\Psi_{2}$ can coexist with incommensurate layer spacings. ${ }^{1,10}$ This is indeed the case in 4-n-heptylphenyl-4'-(4". nitrobenzoyloxy)benzoate $\left(\mathrm{DB} 7 \mathrm{NO}_{2}\right)$, where the $\mathrm{Sm} A_{1}$ phases coexists with an antiferroelectric $\operatorname{Sm} A_{d}$ phase for bulk samples. ${ }^{3,6}$ Safinya and co-workers have shown that only the $\Psi_{2}$ density wave exhibits critical behavior as the transition is approached whereas the $\Psi_{1}$ density wave does not.

In this paper we report the observation of the surface structure of two incommensurate density modulations at the surface of $\mathrm{DB} 7 \mathrm{NO}_{2}$. At a free interface the smectic ordering is enhanced since the centers of mass are fixed and the molecules are orientationally ordered. For instance, $x$-ray reflectivity measurements at the nematic to smectic- $\boldsymbol{A}$ transition in octyloxycyanobiphenyl (8OCB) have shown that at large distances from the surface the smectic order parameter penetrates exponentially into the bulk with the penetration length equal to the parallel correlation length for critical fluctuations. Near the surface, however, the exponential model fails. ${ }^{11,12}$ In the isotropic phase of decylcyanobiphenyl (12CB) the effect of the surface induces a discrete number of smectic layers, where the number of layers depends on temperature. ${ }^{13}$ Recently, Gramsbergen et al. ${ }^{14}$ have shown that in 4-noctyloxyphenyl-4'-(4"-cyanobenzyloxy)benzoate the surface induces short-range antiferroelectric order where the antiferroelectric $q$ vector $q_{1}$ is exactly half the monolayer $q$ vector. For $\mathrm{DB} \mathrm{NO}_{2}$, the ratio of the modulation $q$ vectors is 0.6 ; therefore, the ordering cannot be represented by the simple addition of an antiferroelectric order parameter. Instead, the data require a description of the surface smectic mass density modulation as two incommensurate density waves percolating through one another, as suggested by Prost and Barois. ${ }^{1}$ In contrast to bulk and surface measurements in $8 \mathrm{OCB}$, the incommensurate correlation length at the surface in $\mathrm{DB} \mathrm{NO}_{2}$ does not agree with the bulk values at all temperatures. ${ }^{6}$

The $x$-ray experiments reported here were carried out with a Rigaku rotating anode $x$-ray generator with a $0.3 \times 3.0-\mathrm{mm}^{2}$ fine focus cathode assembly and with a $\mathrm{Ge}(111)$ monochromator tuned for copper $K_{\alpha}$ radiation, $\lambda=1.54 \AA$. Since the surface of the liquid must always remain normal to the earth, the surface spectrometer differs from a standard three-circle spectrometer in that the incident beam must be deflected down in order to set the incident angle. A complete description of the spectrometer instrumentation is published elsewhere. ${ }^{15}$ In Fig. 1 the spectrometer scattering geometry and the reciprocal-space resolution volumes are shown. Since there is no transverse momentum transfer for specular scattering and the angular acceptance of the detector is 


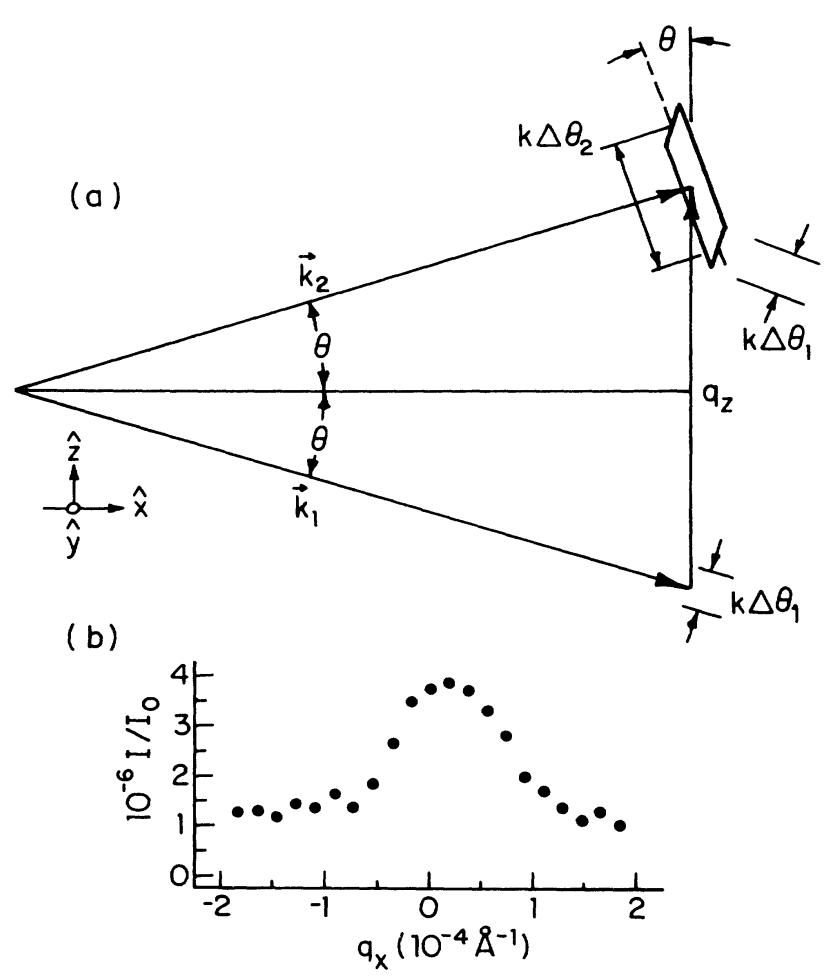

FIG. 1. (a) In-plane scattering geometry where $\left|k_{1}\right|=\left|k_{2}\right|=2 \pi / 1.54 \AA$. Typically $\theta=1.5^{\circ}, \Delta \theta_{1}$ is $0.01^{\circ}$ and $0.02^{\circ}$ for high and low resolution, and $\Delta \theta_{2}=0.08^{\circ}$. The out-ofplane resolution is $0.4^{\circ}$ and $0.8^{\circ}$ for high and low resolution. (b) Typical transverse scan at $q=0.183 \AA^{-1}$. This provides a measure of the transverse resolution function.

larger than the angular spread of the incident beam, $\Delta \theta_{2}>\Delta \theta_{1}$, the spectrometer resolution for surface reflection depends only on $k \Delta \theta_{1}$. In order to optimize the measurement times the data were taken with two spectrometer configurations. The high-resolution configuration has resolution widths which are approximately a factor of 2 smaller along $q_{z}$ and $q_{y}$ than for the lowerresolution configuration. The bulk scattering resolution depends on both the incident and outgoing resolution and was kept as small as possible in order to minimize the signal due to bulk scattering.

The experiment is carried out by measuring the specular reflectivity as a function of the wave vector transfer normal at the surface, $q_{z} \simeq(4 \pi / \lambda) \sin \theta,{ }^{16}$ at various temperatures in the nematic phase of $\mathrm{DB}^{2} \mathrm{NO}_{2}$. Below the critical $q$ vector, $q_{c}=0.0235 \AA^{-1}$, for $\mathrm{DB}^{2} \mathrm{NO}_{2}$ the reflectivity was measured to be $92( \pm 3) \%$. In Fig. 2, the sum of the reflected and scattered signals, for $I / I_{0}$, with $q_{x}=q_{y}=0$ is plotted versus $q_{z}$ at $T=115^{\circ} \mathrm{C}$. The solid line, $R_{F}\left(q_{z}\right)$, which corresponds to the theoretical reflectivity predicted from the Fresnel law of optics for a simple step-function interface falls off as $\left(q_{c} / 2 q_{z}\right)^{4}$ for $q_{z} \gg q_{c}$. Deviations for $q_{z}<q_{c}$ arise since for sufficiently small incident angles the beam spills over the sides of the sample. Over the range $0.018<q_{z}<0.06 \AA^{-1}$ the theoretical reflectivity agrees very well with the displayed data. At larger $q$ vectors there are significant deviations

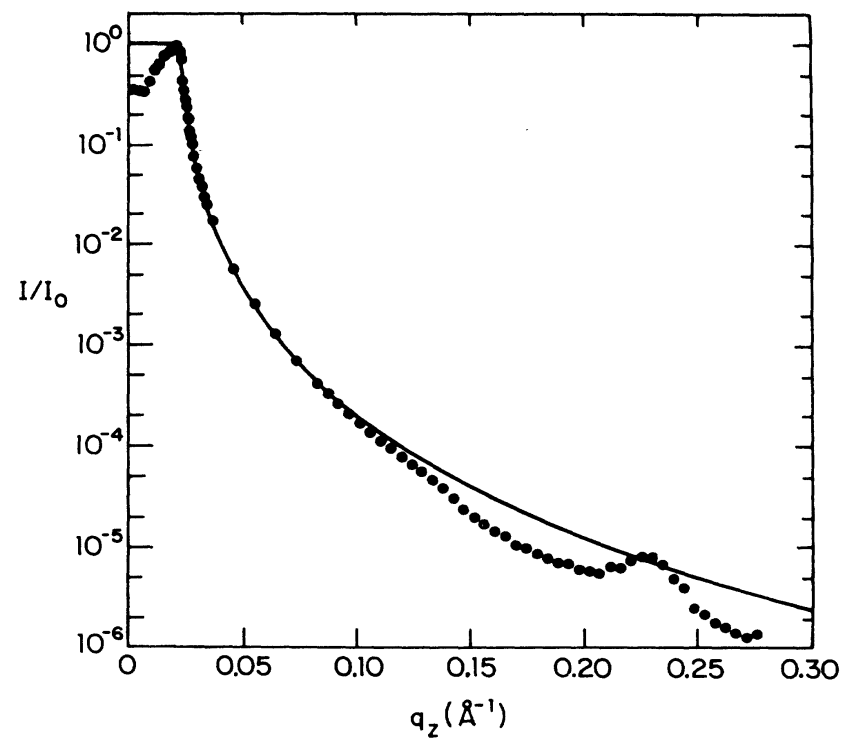

FIG. 2. Scattered intensity normalized to the direct beam $\left(I_{0}\right)$ at $T=115^{\circ} \mathrm{C}$ as a function of $q_{z}$ for the spectrometer tuned to the specular condition (i.e., $q_{x}=q_{y}=0$ ).

in the specular reflectivity from the Fresnel law.

In this experiment it is necessary to separate the bulk scattering from the surface reflectivity. The surface reflectivity is restricted to $q_{x}=q_{y}=0$, whereas the bulk scattering occurs both on and off the $z$ axis. This is clearly evidenced since transverse scans along both $q_{x}$ and $q_{y}$ are composed of a sharp resolution-limited surface peak and a broad bulk peak. Figure 1 (b) shows the resolutionlimited surface peak superimposed on top of a broader diffuse background. For the present study the bulk scattering peak is always broader than the resolution along $q_{x}$, therefore the bulk contribution is measured by a slight displacement along $q_{x} \simeq\left(7 \times 10^{-4}\right) q_{z}$ which is approximately twice the transverse in-plane resolution. The specular scattering is simply the difference between the intensity on and off the $q_{z}$ axis. To account for the effects of a sloping background along $q_{x}$ the average background on both sides of the $q_{z}$ axis is taken.

In Fig. 3 we show $q_{z}$ scans both on and off the $q_{z}$ axis for both resolutions normalized to the direct beam $\left(I_{0}\right)$. At small $q_{z}$, the bulk contribution is several orders of magnitude less than the surface reflection. However, a bulk peak develops at $q_{z} \simeq 0.14 \AA^{-1}$ which corresponds to incommensurate order. Along the $q_{z}$ axis, which measures bulk and surface, the intensity has a local minimum at about this same $q$ vector with an intensity which is only slightly larger than the bulk contribution. Therefore, the specular contribution has an even stronger minimum and appears to go to zero. In Fig. 4, we plot the surface reflectivity using the subtraction technique described in the previous paragraph normalized to $R_{F}\left(q_{z}\right)$, the value obtained from the Fresnel law of optics. We note that since this involves subtracting intensities which are nearly equal at $q_{z} \approx 0.14 \AA^{-1}$ very long counting times were required to obtain reasonable data. Although before subtraction the intensities differ for the two resolutions, as is apparent 


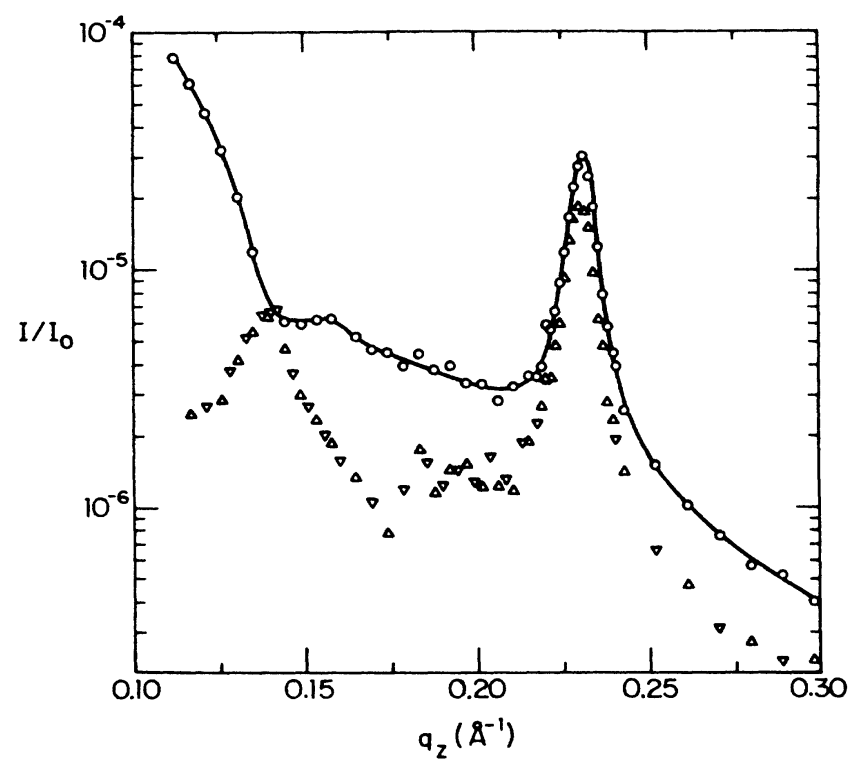

FIG. 3. Scattered intensity normalized to the direct beam $\left(I_{0}\right)$ at $\Delta T=2.0^{\circ} \mathrm{C}$. The points with circles are on the $q_{z}$ axis whereas the triangles are displaced along $q_{x}$ by twice the $\Delta q_{z}$ half width at half maximum. The triangles are displaced in the plus direction and the inverted triangles are displaced in the negative direction.

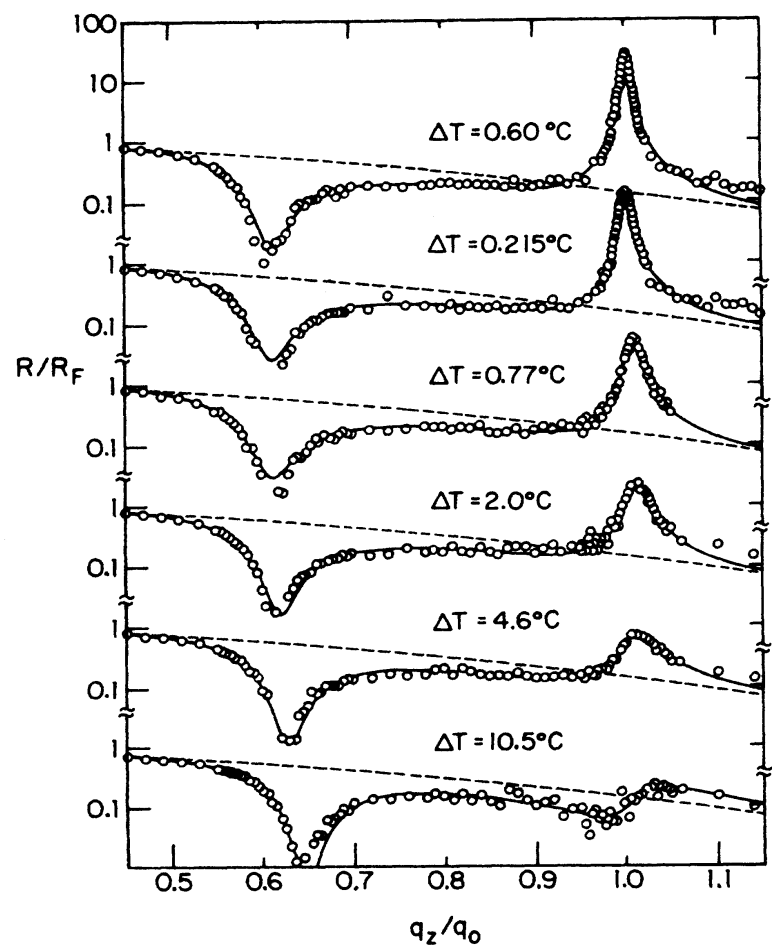

FIG. 4. Surface reflectivity divided by the Fresnel function at six temperatures (circles). The solid lines are the results of fits to Eq. (2), whereas the dashed lines are the error-function contribution to the reflectivity. in Fig. 3 (triangles) at $q_{z} \approx 0.18 \AA^{-1}$, the surface reflectivities in Fig. 4 overlap for both configurations. It is convenient to define $q_{0}=q_{2}\left(T_{N A}\right)=2 \pi / D_{0}=0.229 \AA^{-1}$, where $D_{0}=27.4 \AA$.

The dip at $q_{z}=0.14 \AA^{-1}\left(q_{z} / q_{0}=0.6\right)$ in the surface reflectivity is a consequence of the incommensurate density modulation interfering destructively with the reflection from the liquid-crystal-air interface. On the other hand, the peak at $q_{z}=q_{0}$ is positive since the monolayer modulation constructively interferes with the liquid-crystal-air interface. In Fig. 4 we show representative surface reflectivity data at $T-T_{N A}=0.60,0.215,0.77,2.0,4.6$, and $10.5^{\circ} \mathrm{C}$. From these figures it is clear that the commensurate peak develops as the transition is approached, whereas the incommensurate peak is reasonably temperature independent. This observation is in general agreement with the bulk phase measurements by Safinya and co-workers. ${ }^{6}$

The theoretical expression for the ratio $R\left(q_{z}\right) / R_{F}\left(q_{z}\right)$ in terms of the average electron density gradient along the surface normal is given by

$$
\frac{R\left(q_{z}\right)}{R_{F}\left(q_{z}\right)}=\left|\frac{1}{\langle\rho\rangle} \int_{-\infty}^{\infty}\left\langle\frac{\partial \rho}{\partial z}\right\rangle e^{i q_{z} z} d z\right|^{2}
$$

in the Born approximation limit, i.e., $q_{z} \gg q_{c} \cdot{ }^{17,18}$ We model the density function $\rho(z)$ by a step function smeared by a Gaussian, i.e., an error function, and two independent coexisting sine waves with exponentially penetrating amplitudes,

$$
\begin{aligned}
\rho(z)= & A \operatorname{erf}(z / \sigma)+B_{2} \Theta(z) \sin \left[q_{2}\left(z-z_{2}\right)\right] e^{-z / \xi_{2}} \\
& +B_{d} \Theta(z) \sin \left[q_{d}\left(z-z_{d}\right)\right] e^{-z / \xi_{d}}
\end{aligned}
$$

where $A \approx 1$ and $\Theta(z)$ is a step function at $z=0$. The microscopic surface roughness of simple liquids can also be described by the error-function form. ${ }^{18}$ The second term represents $\operatorname{Sm} A_{1}$ ordering where $q_{2}$ is the commensurate monolayer $q$ vector. Finally, the third term corresponds to the incommensurate $\operatorname{Sm} A_{d}$ phase where $q_{d} \geq \frac{1}{2} q_{2}$ is the incommensurate wave vector.

In Fig. 4 we show the results of these fits as solid lines. Most of the key features of the data, including the amplitudes and widths of the primary peak and the positions of the peaks and dips are well represented by this form. If the amplitude of the two sine waves is set to zero, $R / R_{F}$ reduces to a Gaussian as shown by the dashed lines in Fig. 4. The deviations from the Gaussian behavior are a direct result of constructive and destructive interference from the two sine waves.

Although initially we fit all ten parameters in Eq. (2), we found that $\sigma, z_{d}, B_{d}$, and $\xi_{d}$ did not depend strongly on temperature. Therefore, for the fits presented in Fig. 4 , we have set $\sigma=6.5 \AA{ }^{19} z_{d}=0.77 D_{0}, B_{d}=0.14$, and $\xi_{d}=2.3 D_{0} \quad\left(\xi_{d} q_{d} \simeq 14\right)$. In Table I the parameters obtained from the line-shape fits are summarized. ${ }^{20}$ The incommensurate $q$ vector, $q_{d}$, increases linearly from $0.59 D_{0}$ at the transition to $0.63 D_{0}$ at $10.5^{\circ} \mathrm{C}$ above $T_{N A}$. The most striking feature in Fig. 4 is the dramatic increase in the commensurate correlation length $\xi_{2}$ as the 
TABLE I. Parameters which were obtained by fitting Eq. (2) to the data shown in Fig. 4. The remaining parameters have been fixed at the values $\sigma=6.5 \AA, z_{d}=0.77 D_{0}, B_{d}=0.14$, and $\xi_{d}=2.3 D_{0}$.

\begin{tabular}{lccccc}
\hline \hline$T\left({ }^{\circ} \mathrm{C}\right)$ & $B_{2}$ & $q_{2} / q_{0}$ & $z_{2} / D_{0}$ & $\xi_{2} / D_{0}$ & $q_{d} / q_{0}$ \\
\hline 0.06 & 0.041 & 1.000 & 0.91 & 40 & 0.592 \\
0.215 & 0.037 & 1.000 & 0.90 & 30 & 0.591 \\
0.77 & 0.033 & 1.008 & 0.90 & 20 & 0.589 \\
2.0 & 0.029 & 1.010 & 0.91 & 12 & 0.599 \\
4.6 & 0.022 & 1.008 & 0.85 & 7.7 & 0.610 \\
10.5 & 0.021 & 1.020 & 0.68 & 3.5 & 0.630 \\
\hline \hline
\end{tabular}

transition is approached. Close to the transition the correlation length is nearly resolution limited and accurate deconvolutions with the resolution are extremely difficult with the current geometry. The fits, without deconvolution, are consistent with $\xi_{2}=7.7 D_{0} \quad\left(\xi_{2} q_{2} \simeq 48\right)$ at $T-T_{N A}=4.6^{\circ} \mathrm{C}$ increasing to $\xi_{2}=40 D_{0}\left(\xi_{2} q_{2} \simeq 250\right)$ at $T-T_{N A}$ at $0.060^{\circ} \mathrm{C}$. We note that the width at this temperature is close to being resolution limited. The commensurate modulation peak constructively interferes with the surface reflection and the corresponding phase factor $z_{2}$ is close to zero $\left(z_{2} q_{2}=0.9 \pi \equiv-0.1 \pi\right)$. On the other hand, the incommensurate modulation peak is out of phase with the surface reflection and the phase factor $z_{d} q_{d}=0.45 \pi$. The amplitude of the commensurate modulation, $B_{2}$, increases from 0.021 at $T-T_{N A}=10.5^{\circ} \mathrm{C}$ to 0.041 at $T-T_{N A}=0.06^{\circ} \mathrm{C}$.

The reflectivity measurements, combined with the lineshape analysis, provides an absolute measure of the modulation amplitude for both density waves at the surface. For the nematic phase in $\mathrm{DB} 7 \mathrm{NO}_{2}$ the modulation amplitude for the $\operatorname{Sm} A_{d}$ modulation, $B_{d}=0.14$, is significantly larger than the amplitude of the $\operatorname{Sm} A_{1}$ modulation. It is this large amplitude which is responsible for the intense incommensurate bulk phase scattering. The incommensurate correlation length at the surface, $\xi_{d}^{\text {bulk }} q_{0}=14$, is smaller than the bulk incommensurate correlation length which increases from $\xi_{d}^{\text {bulk }} q_{0}=25$ at high temperatures to $\xi_{d}^{\text {bulk }} q_{0}=50$ at the transition. ${ }^{6,21}$ This is the first instance in which the surface penetration length in a nematic phase differs from the bulk correlation length. We believe that this discrepancy may result from the surface which fixes the relative phase of the two density waves. Our data show that the surface penetration length for the commensurate density wave agrees with the bulk values over the temperature range where the peak widths are broader than our resolution. With higher-resolution data it should be possible to test whether the commensurate surface and bulk lengths agree over a greater temperature range. The $q$ vectors for commensurate and incommensurate ordering at the surface agree absolutely with the bulk values over the entire temperature range.

The characteristic falloff in the reflectivity profile that has been observed at the $q$ vector corresponding to the diverging correlation length for 4-n-octyloxy-4'cyanobiphenyl (8OCB) and butyloxybenzylidene octylaniline (4O.8) is not present at $q_{2}$ in $\mathrm{DB} \mathrm{NO}_{2} \cdot{ }^{22,12}$ For 8OCB and 40.8 this falloff was interpreted as an enhancement of the smectic order at the interface. It is interesting to note that the additional smectic ordering that occurs at the surface of $\mathrm{DB} 7 \mathrm{NO}_{2}$ is at the $\operatorname{Sm} A_{d} q$ vector $\left(q_{d}\right)$ rather than the $\operatorname{Sm} A_{1} q$ vector $\left(q_{2}\right)$. Thus the near-surface structure of $\mathrm{DB} \mathrm{NO}_{2}$ is qualitatively different from that of the previously studied systems. A second features indicating this difference is that the amplitude of the $q_{2}$ smectic modulation increases as the transition is approached in $\mathrm{DB} \mathrm{NO}_{2}$, whereas in the other systems the amplitude is either independent of temperature or decreasing. It is important to note that the precise behavior of the amplitude is model dependent and this surprising effect for the other materials may be an artifact of the model. In any event the near-surface behavior is modeled differently for $\mathrm{DB} \mathrm{NO}_{2}$ than for the other systems.

It is difficult to test whether the form for the density, as presented in Eq. (2) provides a unique representation. For instance, the inclusion of layer smearing provides equally good fits in which only the amplitude parameters are different. A rather attractive possibility is that the surface is really a tilted antiferroelectric $\operatorname{Sm} A_{2}$ which only extends a few layers followed by a $\operatorname{Sm} A_{1}$ phase which penetrates further into the bulk. Of course there may be other alternative models, however, without appropriate theoretical guidance it is difficult to dismiss all of these models.

In summary, the surface of $\mathrm{DB} \mathrm{NO}_{2}$ can be described as the superposition of two incommensurate density waves which emanate from the surface and penetrate exponentially into the bulk. The surface $\operatorname{Sm} A_{d}$ density wave has a periodicity which is less than twice the molecular length and has been parametrized by a form which is independent of temperature except for a slight spatial contraction. This is contrasted with the critical $\operatorname{Sm} A_{d}$ fluctuations for which the correlation length differs by nearly a factor of 2 as the transition is approached. This is the first example in which the penetration length of the surface-induced smectic order is not equal to the bulk correlation length. Although the amplitude and the penetration length of the $\operatorname{Sm} A_{1}$ density wave does appear to diverge in the same manner as for other nematic surfaces, the structure of $R / R_{F}$ on the high wave vector side of the $\operatorname{Sm} A_{1}$ peak is qualitatively different from the nematic surfaces of $8 \mathrm{OCB}, 40.8$, and a number of cyanobiphenyl homologs. The strong asymmetry in the line shape around the layering $q$ vector inherent in most of the previously studied samples was previously interpreted as local surface structure and its absence for $\mathrm{DB} \mathrm{NO}_{2}$ indicates a very dif- 
ferent local surface structure. ${ }^{23}$

The diversity of smectic behavior observed at liquidcrystal surfaces has yet to be explained by a global theoretical picture. Further theoretical work is essential for this class of problems. Synchrotron sources would provide increased fluxes which would allow measurements at larger $q$ vectors and with higher resolution.

This work was supported in part by the National Science Foundation through Grants No. DMR-85-13523 and No. DMR-83-16979 and by the Exxon Research and Engineering Corporation.
1J. Prost and P. Barois, J. Chim. Phys. 80, 65 (1983).

${ }^{2}$ K. K. Chan, P. S. Pershan, L. B. Sorensen, and F. Hardouin, Phys. Rev. Lett. 54, 1694 (1985).

${ }^{3}$ F. Hardouin, G. Sigaud, Nguyen Huu Tinh, and M. F. Achard, J. Phys. (Paris) Lett. 42, L63 (1981).

${ }^{4}$ B. R. Ratna, R. Shashidhar, and V. N. Raja, Phys. Rev. Lett. 55, 1476 (1985).

${ }^{5}$ F. Hardouin, Nguyen Huu Tinh, M. F. Achard, and A. M. Levelut, J. Phys. (Paris) Lett. 43, L327 (1982).

${ }^{6}$ C. R. Safinya, William A. Varady, L. Y. Chiang, and P. Dimon, Phys. Rev. Lett. 57, 432 (1986).

${ }^{7}$ N. H. Tinh, F. Hardouin, and C. Destrade, J. Phys. (Paris) Lett. 43, 1127 (1982).

${ }^{8}$ R. Shashidhar, B. R. Ratna, V. Surendranath, V. N. Raja, S. Krishna Prasad, and C. Nagabhusan, J. Phys. (Paris) Lett. 46, L445 (1985).

${ }^{9}$ Ernest Fontes, Paul A. Heiney, John N. Haseltine, and Amos B. Smith III, J. Phys. (Paris) 47, 1533 (1986).

${ }^{10}$ Jiang Wang and T. C. Lubensky, J. Phys. (Paris) 45, 1653 (1984).

11J. Als-Nielsen, F. Christensen, and P. S. Pershan, Phys. Rev. Lett. 48, 1107 (1983).

12P. S. Pershan and J. Als-Nielsen, Phys. Rev. Lett. 52, 759 (1984).

${ }^{13}$ B. M. Ocko, A. Braslau, P. S. Pershan, J. Als-Nielsen, and M.
Deutsch, Phys. Rev. Lett. 57, 94 (1986).

${ }^{14}$ E. F. Gramsbergen, W. H. de Jeu, and J. Als-Nielsen, J. Phys. (Paris) 47, 711 (1986).

${ }^{15}$ A. H. Weiss, M. Deutsch, A. Braslau, B. M. Ocko, and P. S. Pershan, Rev. Sci. Instrum. 57, 2554 (1986).

${ }^{16}$ The actual momentum transfered in the material is $q_{z}=(4 \pi / \lambda)\left(\sin ^{2} \theta-\sin ^{2} \theta_{c}\right)^{1 / 2}$

${ }^{17}$ M. Born and E. Wolf, in Principles of Optics (Pergamon, Oxford, 1975).

${ }^{18}$ A. Braslau, M. Deutsch, P. S. Pershan, A. H. Weiss, J. AlsNielsen, and J. Bohr, Phys. Rev. Lett. 54, 114 (1985).

${ }^{19}$ Although the value for $\sigma$ that describes the surface roughness obtained here is larger than the values previously obtained for both water (i.e., $\sigma=3.2 \AA$, Ref. 18) and $12 \mathrm{CB}$ (i.e., $\sigma=5.5 \AA$, Ref. 13) we do not believe the difference is statistically significant.

${ }^{20}$ The amplitude $A$ is also varied in the fitting process; however, it varies by less than $10 \%$ over the fitted temperature range, and we do not consider this significant.

${ }^{21}$ The fitted value of $\xi$ in units of $D_{0}$ is smaller than $\xi$ in units of $q_{0}$ by $2 \pi$.

${ }^{22}$ P. S. Pershan, A. Braslau, A. H. Weiss, and J. Als-Nielsen, Phys. Rev. A (to be published).

${ }^{23}$ This feature was also observed in 4-n-octyloxyphenyl-4'-(4". cyanobenzyloxy)benzoate (Ref. 14). 